\title{
A QUANTUM-MECHANICAL ARGUMENT FOR MIND-BODY DUALISM
}

\begin{abstract}
I argue that a strong mind-body dualism is required of any formulation of quantum mechanics that satisfies a relatively weak set of explanatory constraints. Dropping one or more of these constraints may allow one to avoid the commitment to a mind-body dualism but may also require a commitment to a physical-physical dualism that is at least as objectionable. Ultimately, it is the preferred basis problem that pushes both collapse and no-collapse theories in the direction of a strong dualism in resolving the quantum measurement problem. Addressing this problem illustrates how the construction and evaluation of explanatorily rich physical theories are inextricably tied to the evaluation of traditional philosophical issues.
\end{abstract}

From the earliest formulation of the theory, physicists have thought that quantum mechanics has something to teach us concerning the relationship between conscious thought and the physical world. ${ }^{1}$ This view was amplified and given explicit content in 1961 by Eugene Wigner:

Until not many years ago, the "existence" of a mind or soul would have been passionately denied by most physical scientists. ... There are [however] several reasons for the return, on the part of most physical scientists, to the Spirit of Descartes' "Cogito ergo sum" .... When the province of physical theory was extended to encompass microscopic phenomena, through the creation of quantum mechanics, the concept of consciousness came to the fore again: it was not possible to formulate the laws of quantum mechanics in a consistent way without reference to consciousness. Wigner (1961)

While one might take it to be improbable that quantum mechanics provides a compelling argument in favor of a strong Cartesian mindbody dualism, it is presumably at least possible for a physical theory to yield philosophical consequences - even concerning the relationship between mental and physical states. Insofar as they provide our best understanding of the physical world, one should expect good empirical theories both to inform and to be informed by productive philosophical inquiry. More specifically, while I do take Wigner's 
position to be entirely compelling, I will argue that a strong variety of mind-body dualism may indeed be required by quantum mechanics depending on the explanatory demands one places on the theory. ${ }^{2}$ I take this argument and the assessment of its implications to illustrate the inevitable interaction between explanation in our best physical theories and productive philosophical reflection.

In order to see why quantum mechanics was taken to have implications for the philosophy of mind in the first place, it is necessary to understand how the theory works generally and how it treats physical properties and quantities in particular. We will start with the standard Von Neumann-Dirac collapse formulation of quantum mechanics (von Neumann 1955) and Wigner's response to the theory, then extend the discussion to no-collapse formulations of quantum mechanics.

There is a sense in which quantum properties are more geometrical than logical. According to the standard formulation of quantum mechanics, the state of a physical system $S$ is represented by a unitlength vector $[S]_{S}$; and, for each physical property $P$ that one might observe of the system $S$ there is a unit vector $[P]_{S}$ that represents having the property and an orthogonal unit vector $[\text { Not } P]_{S}$ that represents not having the property. While $[P]_{S}$ and $[\text { Not } P]_{S}$ are at right angles to each other, $[S]_{S}$ can in principle point any direction relative to $[P]_{S}$ and $[\text { Not } P]_{S}$. The vector $[S]_{S}$ representing the state of $S$ might, for example, bisect the right angle between $[P]_{S}$ and $[\text { Not } P]_{S}$ and consequently differ from each by $45^{\circ}$. One might represent this state as $[P]_{S}+[\text { Not } P]_{S}$. The standard eigenvalue-eigenstate link is used to interpret such states. This interpretational principle says that a system $S$ determinately has physical property $P$ if and only if $[S]_{S}=[P]_{S}$; and $S$ determinately does not have property $P$ if and only if $[S]_{S}=[\text { Not } P]_{S}$. Hence, if $[S]_{S}$ is at a $45^{\circ}$ angle to both $[P]_{S}$ and [Not $P]_{S}$, then $S$ neither determinately has nor determinately fails to have property $P$. In such a state the system $S$ is said to be in a superposition of having and not having property $P$. Quantum mechanics predicts that a system will typically be in a superposition of having and not having any specified physical property.

Given this, perhaps the most immediate explanitory demand on quantum mechanics is to explain why we never directly observe a system in a superposition of possessing and not possessing a given property; or, put somewhat differently, quantum mechanics should explain why measurements typically yield determinate measurement records. The standard explanation replies on the dual structure of the dynamics in the standard collapse formulation of quantum mechanics: (A) if no 
measurement is made, then a system $S$ evolves continuously according to the linear, deterministic dynamics, which depends only on the energy properties of the system, but (B) if a measurement is made, then the system $S$ instantaneously and randomly jumps to a state where it either determinately has or determinately does not have the property being measured, where the probability of each possible post-measurement state depends on the system's initial state. While this does explain why measurements typically yield determinate physical records, the dual structure of the dynamics and the occurrence of measurement as an undefined primitive term in the theory is at least curious. Albert Einstein, for one, did not believe that this aspect of the theory could be right.

Hillary Putnam tells the story of joining the faculty at Princeton in 1953 and Hans Reichenbach arranging for Putnam to meet Einstein for tea. Rather than complain about the stochastic nature of the standard collapse formulation of quantum mechanics, Einstein was worried about the theory's two dynamics (A) and (B) and the collapse dynamics (B) in particular. Putnam reports: "What he said on that occasion was something like the following: 'Look, I don't believe that when I am not in my bedroom my bed spreads out all over the room, and whenever I open the door and come in it jumps into the corner', (Putnam 2005, p. 10).

In order to see how the theory works and to understand Einstein's worry, consider Einstein $E$ opening his bedroom door and observing the position of his bed $B$. Instead of considering the property of his bed being in the corner of his room, we will consider the property of his bed being in his room at all when he looks for it. Before Einstein opens the door to look, (assuming that his bed's position counts as being unmeasured if Einstein is not look at it), then the linear dynamics (A) predicts that the bed will typically evolve to a state where it is in a superposition of being in Einstein's bedroom and not being in Einstein's bedroom - a state like

$$
[\text { In Room }]_{B}+[\text { Not in Room }]_{B} \text {. }
$$

But as soon as the bed's position is measured, then, by the collapse dynamics (B), its physical state randomly jumps to one where it either is or is not determinately in Einstein's room.

On the eigenvalue-eigenstate link, the indetermincy of the bed's position before being measured is a metaphysical rather than an epistemic indeterminacy - it is in the act of being observed that the bed aquires the property of having a determinate position. It is 
because of this that the physical state must change dramatically on measurement in order to have a determinate measurement record. In other words, it is the eigenvalue-eigenstate link interpretation that requires the dual structure of the dynamics. The linear dynamics (A) explains quantum-mechanical interference phenomena and is thus responsible for the remarkable empirical successes of quantum mechanics; but, given the eigenvalue-eigenstate link, if not for the collapse dynamics (B), the theory would typically fail to predict a postmeasurement state where there is any determinate measurement record whatsoever. It is the standard metaphysics of quantum properties that ultimately leads to the quantum measurement problem.

Suppose that Einstein's mental state supervenes on his physical state, and consider what each dynamical law predicts when Einstein observes the position of his bed. For simplicity, suppose that the bed starts in the pure symmetric state

$[\text { In Room }]_{B}+[\text { Not in Room }]_{B}$

and that Einstein starts in the physical state

$[\text { Ready to record the position of bed }]_{E}$.

According to dynamics (A), if Einstein is a good observer; that is, if he is good at correlating his physical brain record with the position of his bed in those cases where it in fact has a determinate position, the post-measurement state of the composite system after Einstein measures the position of the bed will be

$$
\begin{aligned}
& \text { ([“Bed in room" } \left.]_{E} \text { and }[\text { In Room }]_{B}\right) \\
& \quad+\left([\text { "Bed not in room" }]_{E} \text { and }[\text { Not in room }]_{B}\right) .
\end{aligned}
$$

Here the composite system ends up in a superposition of Einstein recording that the bed is in the room because it is and Einstein recording that the bed is not in the room because it isn't. And on the standard eigenvalue-eigenstate link, Einstein has no determinate measurement record and his bed has no determinate position when in this entangled state. ${ }^{3}$

Under the same initial conditions, dynamics (B) predicts that the state after Einstein looks will be either

$[\text { "Bed in room" }]_{E}$ and $[\text { In room }]_{B}$ 
or

["Bed not in room" $]_{E}$ and [Not in room $]_{B}$.

Here the composite system is either in a state where Einstein determinately records that the bed is in the room because it is or where Einstein determinately records that the bed is not in the room because it isn't; and, given the symmetry of the initial state, each with probability $1 / 2$. In either case, as opposed to the result of dynamics (A), on dynamics (B) Einstein ends up with a fully determinate measurement record and his bed ends up with the fully determinate corresponding position.

Since dynamics (A) and dynamics (B) typically yield different postinteraction states, the logical consistency of quantum mechanics depends on these two laws not describing the evolution of any system at the same time. That is, quantum mechanics can only be said to be consistent if one has strictly disjoint conditions for when each dynamical law obtains. This is the role played by the undefined primitive term measurement in the standard collapse theory dynamics (B) obtains if and only if a measurement occurs. But when exactly is that? One wants to say that the composite system's evolution is correctly described by dynamics (B) in the example above because Einstein's looking constitutes a measurement, but the theory itself does not in any way justify this assumption. It was for the consistency of quantum mechanics generally and more specifically to explain why Einstein's looking constitutes a measurement that Wigner thought a strong Cartesian mind-body dualism was required.

Wigner's (1961) proposed solution to the quantum measurement problem was to replace the standard dual dynamics with a dynamics that explicitly describes the effect of conscious intervention. Wigner's new dynamics says that the state of a physical system typically evolves according to dynamics (A); but when a conscious mind apprehends the state of the system, the system evolves according to dynamics (B), in which case the physical state jumps to one where there is a determinate brain record on which the mental state might be taken to supervene. ${ }^{4}$ This account suggests a strong variety of mind-body dualism since the observer's mental state need not supervene on any aspect of the physical state before apprehending the physical state.

Wigner considered his collapse theory to be the "simplest way out" of the quantum measurement problem (1961, p. 180), and there is a sense in which he was right. Indeed, because of the preferred basis 
problem, there is a sense in which allowing for a strong mind-body dualism provides the simplest way out of the quantum measurement problem for both collapse and no-collapse formulations of quantum mechanics.

Consider how Wigner's collapse theory solves the quantum measurement problem. If Einstein's brain and his bed end up in an entangled superposition, the conscious apprehension of Einstein's brain record of the bed's position by Einstein's nonphysical mind will cause the composite physical system to collapse to a state where Einstein has a determinate brain record and his bed has a determinate position, and this record will be reliable insofar as it was correlated with the position of the bed. One still does not know exactly when collapses occur since one does not know which physical systems are associated with conscious minds. But, as Wigner noted, this is, at least in principle, a matter for empirical investigation - whatever physical systems we find to cause collapses would, in the context of Wigner's theory, be associated with a conscious mind. One might then compare these systems against those that are typically taken to be associated with conscious minds and check for agreement between Wigner's theory and our intuitions concerning which entities are conscious. The problem is that it is virtually impossible in practice to do those experiments that would empirically evaluate Wigner's claim that conscious entities and only conscious entities cause collapses. ${ }^{5}$

There is a sense, however, in which this survey of Wigner's theory misses the point entirely. Insofar as Wigner's theory solves the measurement problem, it does so not by correctly predicting when collapses occur but by stipulating that they do occur and precisely when needed to explain the presence of the determinate measurement record of which the observer is conscious. The only current empirical fact that would be explained by postulating conscious minds that cause collapses in the physical state is the determinateness of each conscious observer's measurement record precisely when such a record is needed. Of course, Wigner's explanation of the determinateness of measurement records is ad hoc since it is exactly those observers who are conscious of determinate records who are stipulated to cause the collapses that generate those records. But this is also why his theory solves the quantum measurement problem. The collapse of the quantum-mechanical state was only ever needed to explain how observers get the determinate measurement results that they do. Wigner's proposal is the simplest way out of the quantum measurement problem for a collapse theory because it directly 
guarantees determinate physical measurement records for whatever entities are in fact conscious precisely when needed.

The causal intervention of nonphysical minds in physical processes is not a necessary requirement for a collapse formulation to explain determinate measurement records. GRW and other stochastic localization formulations of quantum mechanics can also make a claim to solving the quantum measurement problem. ${ }^{6}$ A stochastic localization theory works by selecting a preferred position-like quantity $R$, then replacing the dual dynamics (A) and (B) with a single stochastic dynamics that is nearly linear for simple microscopic systems but pushes the state of more complex macroscopic systems toward a randomly selected state where the value of $R$ is determinate. Such a theory explains determinate measurement records if and only if the value of the physically preferred quantity $R$ in fact determines the values of measurement records - which is presumably the case if and only if observers' beliefs concerning measurement outcomes in fact supervene on the value of $R$.

Having to select a single just-right physical quantity here for the theory to make determinate is an example of the preferred basis problem. If an observer's mental state does not in fact supervene on the value of $R$ (the rough position of the center of mass of a macroscopic object in GRW), then the observer will not get a determinate measurement record on the theory. ${ }^{7}$ The cost of avoiding mind-body dualism in a collapse theory here is that one cannot directly guarantee determinate, epistemically accessible measurement records. In other words, Wigner's theory buys the guarantee that there will always be determinate measurement records exactly when any conscious observer needs one by embracing mind-body dualism. This is not just the simplest way to get determinate mental records in a collapse theory, it is the only way that is guaranteed to work insofar as there may in fact be no physical quantity $R$ on which mental states can be taken to supervene.

While it may be the most direct way to get determinate physical measurement records in a collapse formulation of quantum mechanics, there is also a sense in which Wigner's theory is nevertheless metaphysically extravagant: if one is willing to allow for mind-body dualism in order to get a direct explanation of determinate measurement records, then one does not need to propose a collapse of the quantum-mechanical state at all. Since we have never had direct empirical evidence for the collapse of the quantummechanical state (see endnote 5), the only explanatory role of the collapse dynamics is to explain those determinate measurement 
records we have in situations where the linear dynamics and eigenvalue-eigenstate link would not predict determinate records. But if an observer has a nonphysical mind with an always determinate mental state, then this determinate state already explains the determinate result of a measurement regardless of whether or not there is a collapse of the quantum-mechanical state. One just needs to make sure that the mental dynamics one adopts yields the right statistical distribution for the observer's determinate mental records. This is the idea behind Albert and Loewer's (1988) single- and many-minds formulations of quantum mechanics.

For the sake of economy, we will consider only Albert and Loewer's single-mind formulation of quantum mechanics here. ${ }^{8}$ The single-mind theory is a no-collapse formulation of quantum mechanics: dynamics (A) always correctly describes the evolution of every physical system and there are never collapses of the quantummechanical state. Consequently, Einstein and his bed end up in an entangled state like

$$
\begin{aligned}
& \left.\left([\text { ["Bed in room" }]_{E} \text { and [In Room }\right]_{B}\right) \\
& \quad+\left([\text { "Bed not in room" }]_{E} \text { and }[\text { Not in room }]_{B}\right) .
\end{aligned}
$$

But on the single-mind formulation quantum mechanics Einstein also has a nonphysical mind that always has a determinate mental state. His mental state determines his measurement result; and, on the mental dynamics given by Albert and Loewer, it randomly evolves to a particular measurement result with the standard quantum probabilities. So, while Einstein does not have a determinate physical record, he will always have a determinate mental record of either seeing the bed in his room or a determinate mental record of not seeing the bed in his room.

Since there is no collapse of the quantum-mechanical state here, this way out of the quantum measurement problem is arguably even more direct and simple than Wigner's collapse theory; but, like Wigner's theory, Albert and Loewer's single-mind formulation of quantum mechanics requires that one commit to a strong variety of mind body dualism - strong because the state of an observer's mind cannot be taken to supervene on her physical state. This lack of mental supervenience has the consequence that an observer's mind must also have a transtemporal identity insofar as one can explain her ability to reliably remember her own past measurement results. ${ }^{9}$

While the commitment to mind-body dualism required by the single-mind formulation is unattractive, it is arguably preferable to 
the variety of mind-body dualism suggested by Wigner's collapse theory. Since Wigner's minds need not supervene on the physical state of any system, they are potentially at least as troubling as Albert and Loewer's minds. But while the single-mind theory can be understood as epiphenomenal, where the physical state evolves independently of mental states and the minds are just along for the ride, on Wigner's theory, the evolution of the physical state is affected by what the minds apprehend. The combination of a lack of mental supervenience and the causal efficacy of minds makes Wigner's commitment to mind-body dualism look particularly strong.

Given the specific formulations of the theory that we have considered so far, one might wonder to what extent, if at all, such a strong variety of mind-body dualism is required by quantum mechanics. The short answer is that it depends on what sort of explanations one wants. Given sufficiently strong explanatory demands, one can argue that quantum mechanics requires a commitment to a strong variety of mind-body dualism. But even if one weakens these explanatory demands, while one can avoid an explicit commitment to mind-body dualism, it is difficult to avoid a commitment to some variety of metaphysical pluralism. While I take no-collapse formulations of quantum mechanics to be more plausible than collapse theories, largely because we have never had direct empirical evidence for the collapse of any physical system, avoiding a commitment to a strong variety of metaphysical pluralism is even more difficult for no-collapse theories.

The following four explanatory constraints are sufficient to make a strong variety of mind-body dualism necessarily for any no-collapse formulation of quantum mechanics that satisfies them. We will start with this argument, then examine how one or more of these constraints might be weakened.

No Collapse: The linear dynamics provides a complete and accurate description of the evolution of the physical state for all systems at all times.

State Completeness: The standard quantum-mechanical state is a complete and accurate representation of the physical state.

No Branching: The measurement interaction between an observer and a physical system yields at most a single observer with at most a single measurement record.

Empirical Consistency: If an object system is initially in a superposition of states corresponding to different values of the measured 
observable, then it is possible for any of the values represented in the initial state to be realized as the measurement result.

Consider a system that is initially in a superposition of states where the observable being measured has incompatible determinate values. Given that there is no collapse of the quantum-mechanical state, the linear dynamics predicts that, after a measurement interaction, a good observer will end up in an entangled superposition of recording mutually incompatible measurement results. Since there is no branching to yield different post-measurement observers with different determinate measurement records, there will be only one post-measurement observer with one measurement record. By state completeness, the post-measurement quantum-mechanical state is a complete representation of the physical state. And, by the linearity of the dynamics and empirical consistency, each of the records represented in the post-measurement state are in fact possible measurement results. But, under these conditions, there is nothing in the complete postmeasurement physical state that selects one of the possible records as actual. So insofar as the observer has a determinate measurement record, its value must be must be determined by something that is not determined by the complete physical state. The commitment to such a parameter is a commitment to a strong variety of mind-body dualism insofar as it is the value of this nonphysical parameter, not the complete physical state, that determines the observer's experience and beliefs concerning the outcome of the measurement. ${ }^{10}$

The structure of this argument can be seen in the Einstein's bed example. Suppose that Einstein looks for the bed initially in the symmetric state

$[\text { In Room }]_{B}+[\text { Not in Room }]_{B}$.

If there is no collapse and if Einstein is a good observer in the determinate bed-position cases, then the linear dynamics predicts that the post-measurement state will be the entangled superposition

$$
\begin{aligned}
& \text { (["Bed in room" } \left.\left.]_{E} \text { and [In Room }\right]_{B}\right) \\
& \quad+\left([\text { "Bed not in room" }]_{E} \text { and }[\text { Not in room }]_{B}\right) .
\end{aligned}
$$

If there is no branching, then there is only one post-measurement copy of Einstein with one measurement record. By state completeness the entangled post-measurement superposition is the complete physical state. And by empirical consistency each term in the superposition represents a possible measurement outcome. But there is nothing in 
the complete entangled physical state above that determines which one of the possible records obtains. Hence, if Einstein's belief concerning the value of the measurement record is determined by the state of the world at all, it must be determined by a nonphysical feature of this state. ${ }^{11}$

Insofar as a commitment to a strong mind-body dualism is objectionable, and such a commitment is objectionable if one is concerned to have a purely physical explanation for the existence of physical measurement records, it is natural to consider each of the above explanatory constraints with an eye to which might be sacrificed. Choosing one or more of these constraints to sacrifice is a matter of weighing the explanatory cost of the sacrifice against the potential explanatory return. The costs are primarily in what can be explained by the theory and the returns are in the plausibility of the explanations.

The no collapse constraint rules out the standard von NeumannDirac collapse formulation of quantum mechanics as providing a satisfactory explanation of determinate measurement records. This particular consequence of the constraint is unobjectionable since, because of the quantum measurement problem, the standard collapse formulation of quantum mechanics clearly does not provide a satisfactory explanation of determinate measurement records. But this explanatory constraint also rules out stochastic localization theories. While one should not dismiss such theories out of hand, it is also unclear how much one can expect to gained since, as discussed earlier, it is unclear that such theories in fact explain our determinate measurement records. Like Bohmian mechanics, stochastic localization theories seek to explain determinate measurement records by way of a particular determinate physical observable on which mental states must supervene in order for the theory to explain determinate measurement outcomes. More specifically, one can explain determinate mental records if and only if only if determinate mental records in fact supervene on the preferred position-like physical quantity $R$, which, in the case of GRW, is the roughly determinate positions of the centers-of-mass of macroscopic objects. The preferred basis problem encountered by such collapse theories is immediately solved by adopting Wigner's proposal that it is the conscious intervention of an observer that makes exactly the right physical quantity determinate that would explain the observer's determinate measurement record. But the cost of this solution for collapse theories is a strong variety of mind-body dualism.

The state completeness constraint prevents one from explaining determinate measurement records by appealing to the value of some 
extra physical parameter. This constraint rules out hidden-variable theories like Bohmian mechanics as providing satisfactory explanations of determinate measurement records. ${ }^{12}$ But dropping this constraint does not necessarily eliminate the threat of some variety of dualism. While Bohm's theory does not require a strong mind-body dualism, it does involve a dualistic commitment. In a hidden-variable theory like Bohmian mechanics the physical state has two components: the standard quantum-mechanical state that evolves in the usual unitary way and the always-determinate hidden-variable, particle position in Bohm's theory, that evolves in a way that depends on the evolution of the quantum-mechanical state. One can explain determinate measurement records with this Bohmian physical-physical dualism if and only if determinate particle positions in fact produce determinate measurement records. But again, just as in the stochastic localization theories considered earlier, it is not at all clear that this should be true in general. ${ }^{13}$ The preferred-basis problem for Bohmian mechanics involves the difficulty in explaining why determinate mental records should be expected to supervene on determinate positions. ${ }^{14}$ And, for both collapse and no-collapse formulations of quantum mechanics, addressing the preferred-basis problem pushes in the direction of a commitment to mind-body dualism.

The no branching constraint requires there to be at most one measurement outcome. This constraint rules out many-worlds formulations of quantum mechanics where every quantum-mechanically possible measurement outcome is in fact realized in some world. ${ }^{15}$ On such a theory, it is the fact that there are many fully realized post-measurement copies of an observer, each with a determinate measurement record, that explains why an observer should expect to get a determinate measurement record. But if one does not want to be committed to a strong variety of mind-body dualism, then one would presumably find such a many-worlds pluralism similarly unattractive. $^{16}$

The empirical consistency constraint is difficult to drop since it follows from requiring a satisfactory formulation of quantum mechanics to be empirically adequate. Our best empirical evidence is that the actual measurement result sometimes corresponds to one term in the initial state of the object system (written in the basis corresponding to the measured observable) and sometimes to another; so each term in this expansion of the initial state with a nonzero coefficient must represent a possible measurement result. This constraint follows from empirical adequacy since as the latter requires that the probability of each such possible result is given by 
the norm-squared of the coefficient on the corresponding term in the initial state.

Since the empirical consistency constraint is needed for the empirical adequacy of the theory and since dropping the no branching constraint leads to a metaphysical pluralism that is at least as disturbing as mind-body dualism, it seems to me that the most attractive options for avoiding a commitment to mind-body dualism are either to give up the no collapse constraint or the state completeness constraint. There are, I think, at least two good reasons for preferring to keep the no-collapse constraint and to drop the state completeness constraint. The first is the recurring point that we have no direct empirical reason for supposing that the linear dynamics (A) is ever violated. The second is that the linear dynamics (A) can be translated into a form that is compatible with the constraints of relativity more readily than the collapse dynamics (B). ${ }^{17}$ But, to better understand the costs, let's consider how one might address the preferred basis problem in a no-collapse hidden variable theory.

If one drops the state completeness constraint, one can construct a hidden-variable theory by adding an always-determinate physical parameter to the standard quantum-mechanical state that determines the values of measurement records. If one adds determinate particle positions, then one ends up with a hidden-variable theory like Bohmian mechanics where one has explained determinate measurement records if and only if they can be taken to always supervene on particle positions. Whatever else one might say about the prospects for this, it will, however, prove impossible to accomplish in the context of a quantum field theory where there is nothing directly analogous to particular position. ${ }^{18}$ In the effort to guarantee that one has made an observer's measurement records determinate, one might add a physical hidden-variable $Q$ to the standard quantum-mechanical state such that $Q$ is that physical quantity on which mental records in fact supervene, whatever this happens to be. ${ }^{19}$ The quantum-mechanical state evolves in the usual linear way, and an auxiliary dynamics describes the evolution of the determinate value of $Q$ just as the auxiliary dynamics describes the evolution of determinate particle positions in Bohmian mechanics. The value of $Q$ plays the role of the determinate mental states in the single-mind theory by guaranteeing determinate mental records, but here one seeks to exchange mind-body dualism for a variety of physical-physical dualism.

This $Q$-theory solves the quantum measurement problem if and only if there is a single physical quantity $Q$ on which all mental records in fact supervene. Simply stipulating that there is a just-right 
physical quantity $Q$ that is always determinate and in fact determines all mental states looks more than a little ad hoc (see endnote 18). Moreover, since one is left with a hidden-variable theory where there are two very different types of physical parameters, the quantummechanical state and the determinate physical quantities, each with their own dynamical laws, one has arguably not altogether escaped from committing to a strong metaphysical dualism.

At this point one might wonder why the preferred basis problem is not solved once and for all by making every physical quantity simultaneously determinate and adding all of these determinate physical quantities to the standard quantum-mechanical state, each with its own dynamics. There would then be no need to select a single just-right physical quantity to make determinate since the mental state of an observer presumably always supervenes on some physical quantity. It turns out that every physical quantity can be made simultaneously determinate and dynamical laws be given so that they each exhibit the standard quantum statistics, but, because of the Kochen-Specker theorem, this involves significant conceptual costs and ultimately requires a variety of physical pluralism that arguably rivals mind-body dualism in implausibility. This option is considered in some detail elsewhere. ${ }^{20}$

Given the embarrassment of having to select a single just-right preferred physical quantity $Q$ as always determinate, one might, after all, opt for mind-body dualism as a direct solution to the measurement problem. A mind-body dualism like that of the single-mind theory does not involve just-right stipulations concerning what physical properties are in fact determinate. Moreover, it arguably provides the most direct explanation possible of determinate measurement records since mental states are guaranteed to be determinate without having to bear a special supervenience relation to a privileged physical hidden-variable. In any case, the choice between something like the single-mind theory and the $Q$-theory amounts to a choice between competing hidden-variable theories, each with its own sort of dual structure: with the single-mind theory, the dualism is mental-physical and the hidden-variable that is added to ensure determinate records is the nonphysical mental state of each observer; with the $Q$-theory, the dualism is physical-physical and the hidden variable is the single privileged physical quantity $Q$ that has its own special dynamics and on which determinate mental records are supposed to supervene.

The reason that both theories involve a commitment to some sort of dualism is simple. Given how the quantum-mechanical state evolves 
on the linear dynamics alone, the only way to have determinate measurement records without branching is to add the determinate records to the state description by introducing a new parameter. The variety of dualism that a no-collapse formulation of quantum mechanics requires is between the quantum-mechanical state and the determinate measurement record. One can either posit a physical quantity $Q$ whose value determines mental records or, more directly, one can simply stipulate that an observer always has determinate mental records and avoid the intermediate crutch of introducing a new physical hidden variable. But either way one is embracing a sort of dualism involving significant costs in explanatory plausibility.

Since a given mental record will typically not supervene on the observer's quantum-mechanical state, the single-mind theory, which may provide the most direct way solving the preferred basis problem in a no-collapse theory, requires a strong mental-physical dualism. In one sense the directness of the single-mind theory is a virtue, but it is also this directness that makes it look ad hoc. And the hidden-variable $Q$-theory is arguably no more compelling. Since the value of $Q$ will typically not supervene on the quantum-mechanical state, such a hidden variable involves a variety of physical-physical dualism analogous to the mental-physical dualism of the single-mind theory. And insofar as the only explanatory role of $Q$ is to provide something determinate on which mental states might supervene, this physicalphysical dualism is presumably just as objectionable as the mentalphysical dualism of the single-mind theory. Indeed, there is a sense in which this physical-physical dualism only differs from the mentalphysical dualism by our calling $Q$ a physical rather than a mental parameter. Put another way, it is unconvincing to refer to the quantity $Q$ as a physical quantity when its only explanatory role is to provide something one which mental states might supervene and when it is stipulated to be whatever must be determinate in order for mental states to be determinate. There may be a difference in the explanatory flavor of the single-mind and the $Q$-theory, but they both end up embracing an ad hoc dualism for precisely the same explanatory purpose.

The upshot is that just as the preferred basis problem makes Wigner's theory the simplest and most direct solution to the quantum measurement problem for collapse theories, the preferred basis problem arguably makes the single-mind theory the simplest and most direct solution of the measurement problem for no-collapse theories. This is the sense in which addressing the preferred basis problem pushes both collapse and no-collapse formulations of quantum 
mechanics in the direction of a commitment to mind-body dualism. It is unfortunate that in each case the simplest and most direct resolutions of the quantum measurement problem are also blatantly ad hoc. Insofar as avoiding ad hoc explanations is a standing explanatory constraint, it seems to me that neither of these proposals is ultimately satisfactory. But that is not to suggest that there are currently alternative that are clearly better in every salient way.

While it remains unclear how best to address the preferred basis problem, there are two natural morals one might draw here. A physical moral is that while quantum mechanics certainly does not require a commitment to a strong variety of mind-body dualism, depending on one's explanatory demands, it may favor one, and any no-collapse formulation of quantum mechanics may require a variety of metaphysical pluralism that is at least as objectionable. A methodological moral is that the construction and evaluation of explanatorily rich theories are inextricably tied to the evaluation of traditional philosophical issues.

\section{ACKNOWLEDGMENTS}

I should like to thank David Malament, Craig Callender, Chris Smeenk, Henry Stapp, and especially Ian Thompson for helpful comments on an earlier draft of this paper.

\section{NOTES}

${ }^{1}$ Niels Bohr is a particularly influential example. Bohr's views on the relationships between thought and the world were developed around his notion of phenomena. See Bohr's (1949) account of his discussions with Einstein concerning quantum mechanics and his more general discussion (1960) concerning knowledge in the context of modern physics. While Wigner (1961, p. 174) quotes Bohr (1960), it is unlikely that Bohr would have liked Wigner's (1961) proposal since, I believe, Bohr would have considered it to lack the subtlety of his own interpretation of quantum mechanics. One might, however, argue that what Wigner's proposal may lack in subtlety, it makes up for in clarity.

2 The argument for dualism discussed here has a different structure from Wigner (1961) arguments, which is understandable given the different aims. Wigner's was concerned with finding what he considered to be the simplest way out of the quantum measurement problem in the standard collapse formulation of quantum mechanics. Here I am concerned to explain how the preferred basis problem pushes both collapse and no-collapse theories in the direction of a commitment to mind-body dualism.

${ }^{3}$ It does not matter how subtle or complex Einstein's brain may be or exactly what the supervenience relation between his mental state and physical state is here: if he 
has the disposition to report that the bed is in the room when it is and to report that the bed in not in the room when it isn't, then by the linearity of dynamics $(A)$, Einstein will end up in an entangled superposition of having the disposition to report that the bed is in the room and having the disposition to report that the bed is not in the room in this state. If one were to stipulate that Einstein's beliefs always supervene on his dispositions to report, then one might take this to entail that Einstein would also have a superposition of beliefs in this state. While there is much to say about the possibility of superposed mental states, the possibility of such states resulting from measurement interactions like the one described here is typically dismissed as being incompatible with our direct introspective knowledge. Discussions of the possibility of superpositions of mental states are often found in connection with discussion of the bare theory (See, for example, Albert 1992, p. 112-125).

${ }^{4}$ Hodgson (1991) provides perhaps the most detailed reconstruction of Wigner's proposal.

${ }^{5}$ There has never been any direct empirical evidence for the quantum mechanical state of any physical system ever collapsing - that is, every physical system has always proven to follow the linear dynamics (A) insofar as we can control and isolate the system well enough to determine empirically whether it is following dynamics (A) or dynamics (B). A proponent of Wigner's theory would almost certainly reply that this is because we have not yet done the right experiments. The problem is that it is in practice extremely difficult to directly detect the collapse of the state of a macroscopic system because interactions between the system and its environment will typically destroy precisely those interference effects that would distinguish dynamics (A) from dynamics (B). Those macroscopic systems that are the best candidates for being associated with conscious minds are virtually impossible to isolate sufficiently from their environments. See Albert (1992, p. 88-92) concerning the difficulty in detecting macroscopic collapses.

6 The GRW formulation of quantum mechanics was proposed by Ghirardi et al. (1986). There are more subtle stochastic localization theories, but they all rely on a dynamics that is nearly linear for simple systems but puts complex systems in states close to determinate positions.

${ }^{7}$ Albert (1992, p. 104-111) explicitly describes an observer whose beliefs would not be made determinate by a GRW collapse.

${ }^{8}$ See Barrett (1999, p. 185-220) for a detailed discussion of both theories.

${ }^{9}$ While Albert and Loewer argue that their many-minds formulation of quantum mechanics allows for an observer's mental state to supervene on her physical state, as discussed in Barrett (1995) and (1999), the sort of global supervenience one gets here is very weak. More specifically, precisely the same lack of supervenience one finds in the single-mind theory is also found for each of an observer's minds on Albert and Loewer's many-minds theory.

${ }^{10}$ Among the background assumptions here is the assumption that a satisfactory explanation of a determinate measurement record requires the complete state of the world to determine the value of the record. This requirement can be thought as following from a more general explanatory requirement that all matters of fact should be determined by the complete state. Such assumptions are so basic as to have the flavor of definitions.

11 Since each term in the post-measurement state, written in the determinate record basis, represents an empirically possible measurement result, the empirical 
consistency condition prevents one from appealing to any lack of symmetry in the post-measurement state to select the term that represents the measurement result that is in fact realized. Because of the symmetry of the post-measurement physical state in this particular example, however, it follows directly from state completeness that the complete physical state cannot determine the measurement result since there is nothing in the structure of the mathematical representation of the physical state that might be used to select one term over the other.

12 Bohmian mechanics is the hidden-variable theory proposed by Bohm (1952). See Barrett (1999, p. 127-48) for a description of Bohmian mechanics.

13 Albert describes how Bohmian mechanics might fail to provide an observer with determinate mental records (1992, p. 170-76). This example is similar to his example of how GRW might fail to explain determinate measurement records.

${ }^{14}$ See Barrett (1999, p. 144-146) for a discussion of the preferred basis problem in Bohmian mechanics.

15 Something like the splitting-world formulation of quantum mechanics is assumed in the present discussion. Such a formulation also encounters other, arguably more serious, problems (Barrett 1999, p. 149-184).

16 For the sake of completeness, the way that the no branching constraint is formulated here, it also rules out Albert's bare theory (1992, p. 112-125) insofar as the bare theory predicts that a measurement interaction typically yields none of the determinate measurement outcomes represented in the post-measurement superposition then explaining the appearance of record determinateness as an illusion. But since the cost of embracing the bare theory is undermining the assumptions that ground empirical inquiry generally, the bare theory presumably does not provide a good argument for dropping the no branching constraint. See Barrett $(1994,1996)$ for descriptions of the virtues and vices of the bare theory.

17 See Barrett (2005a) for a recent discussion of the difficulty getting a formulation of quantum mechanics that is compatible with the constraints of relativity.

18 It could happen that good physical reasons are someday found for choosing a preferred physical quantity over all others as determinate. If this quantity were such that one could take measurement records as in fact supervening on it, then this would solve the preferred basis problem. It is sometimes argued by proponents of Bohm's theory that particle position is just such a quantity. Such arguments are, however, ultimately not compelling since particle position is not an available option in quantum field theories. Indeed, in this context, there is arguably no analogous option. Finally, since it cannot be the right quantity to make determinate in a quantum field theory, insofar as we are committed to such a theory and insofar as one finds the arguments in favor of taking particle position as privileged to be compelling, one has, it seems to me, only served to lower the likelihood that one's physical intuitions might serve to reliably decide the issue.

19 For more detailed discussions of the single-thread or $Q$-theory see Barrett (1999, p. 204-20 and (2005b).

20 The Kochen and Specker theorem (1967) shows that one can only make every physical quantity determinate and still have the standard quantum mechanical statistics if one gives up the very functional relationships between physical quantities that are used to define the physical quantities classically. See Barrett (2005b) for a discussion of this theorem and how one might understand the constraint it places on hidden-variable theories. 


\section{REFERENCES}

Albert, D. Z.: 1992, Quantum Mechanics and Experience, Harvard University Press, Cambridge, MA.

Albert, D. Z. and B. Loewer: 1988, 'Interpreting the Many Worlds Interpretation', Synthese 77, 195-213.

Barrett, J. A.: 1994, 'The Suggestive Properties of Quantum Mechanics without the Collapse Postulate', Erkenntnis 41, 233-252.

Barrett, J. A.: 1995, 'The Single-Mind and Many-Minds Formulations of Quantum Mechanics', Erkenntnis 42, 89-105.

Barrett, J. A.: 1996, 'Empirical Adequacy and the Availability of Reliable Records in Quantum Mechanics', Philosophy of Science 63, 49-64.

Barrett, J. A.: 1999, The Quantum Mechanics of Minds and Worlds, Oxford University Press, Oxford.

Bohm, D.: 1952, 'A Suggested Interpretation of Quantum Theory in Terms of 'Hidden Variables', Parts I and II, Physical Review 85, 166-179 and 180-193.

Barrett, J. A.: 2005a, 'Nonlocal Correlations and Explanation in Relativistic Quantum Mechanics', Forthcoming in Philosophy of Science.

Barrett, J. A.: 2005b, 'The Preferred-Basis Problem and the Quantum Mechanics of Everything', Forthcoming in: British Journal for the Philosophy of Science.

Bohr N.: 1949, 'Discussion with Einstein on Epistemological Problems in Atomic Physics', in Schilpp, P. A. (ed.), Albert Einstein: Philosopher-Scientist, pp. 200-241. Reprinted in Wheeler and Zurek (eds.) 1983, 9-49.

Bohr, N.: 1960, Atomic Physics and Human Knowledge, John Wiley \& Sons, New York.

Ghirardi, G. C., A. Rimini and T. Weber: 1986, 'Unified Dynamics for Microscopic and Macroscopic Systems', Physical Review D 34, 470.

Hodgson, D.: 1991, The Mind Matters: Consciousness and Choice in a Quantum World, Clarendon Press, Oxford.

Kochen, S. and E. P. Specker: 1967, 'The Problem of Hidden-variables in Quantum Mechanics', Journal of Mathematics and Mechanics 17, 59-87.

Putnam, H.: 2005, 'A Philosopher Looks at Quantum Mechanics (Again)', Forthcoming in: British Journal for the Philosophy of Science.

von Neumann, J.: 1955, Mathematical Foundations of Quantum Mechanics (Princeton University Press, Princeton). Translated by R. Beyer from Mathematische Grundlagen der Quantenmechanik, Springer, Berlin, 1932.

Wheeler, J. A. and W. H. Zurek (eds.): 1983, Quantum Theory and Measurement, Princeton University Press, Princeton.

Wigner, E. P : 1961, 'Remarks on the Mind-Body Question', Originally published in The Scientist Speculates, Good, I. J. (ed.), Heinemann, London, pp. 284-302. Reprinted in Wheeler and Zurek (eds.) (1983, 168-181).

University of California

765 Social Science Tower, Mail Code: 5100, Irvine

CA, 92697, USA

E-mail: jabarret@uci.edu 